\title{
Giraffe translocation population viability analysis
}

\author{
Derek E. Lee ${ }^{1, *}$, Elmar Fienieg ${ }^{2}$, Cock Van Oosterhout ${ }^{3}$, Zoe Muller ${ }^{4}$, \\ Megan Strauss ${ }^{5}$, Kerryn D. Carter ${ }^{6}$, Ciska P. J. Scheijen ${ }^{7}$, Francois Deacon ${ }^{7}$ \\ ${ }^{1}$ Biology Department, Pennsylvania State University, Muller Laboratory, State College, PA 16801, USA \\ ${ }^{2}$ European Association of Zoos and Aquaria, Plantage Kerklaan 40, 1018 CZ Amsterdam, The Netherlands \\ ${ }^{3}$ School of Environmental Sciences, University of East Anglia, Norwich Research Park, Norwich NR4 7TJ, UK \\ ${ }^{4}$ School of Biological Sciences, University of Bristol, Life Sciences Building, 24 Tyndall Ave, Bristol BS8 1TQ, UK \\ ${ }^{5}$ Wild Nature Institute, 15 North Main Street \#208, Concord, NH 03301, USA \\ ${ }^{6}$ Elephant Connection, Mwandi, Western Province, Zambia
}

${ }^{7}$ Animal, Wildlife and Grassland Sciences, University of the Free State, Nelson Mandela Drive, Bloemfontein, 9301, South Africa

\begin{abstract}
Most populations of giraffes have declined in recent decades, leading to the recent IUCN decision to upgrade the species to Vulnerable status, and some subspecies to Endangered. Translocations have been used as a conservation tool to re-introduce giraffes to previously occupied areas or establish new populations, but guidelines for founding populations are lacking. To provide general guidelines for translocation projects regarding feasibility, we simulated various scenarios of translocated giraffe populations to identify viable age and sex distributions of founding populations using population viability analysis (PVA) implemented in Vortex software. We explored the parameter space for demography and the genetic load, examining how variation in founding numbers and sex ratios affected $100 \mathrm{yr}$ probability of population extinction and genetic diversity. We found that even very small numbers of founders ( $\mathrm{N} \leq 10$ females) can appear to be successful in the first decades due to transient positive population growth, but with moderate population growth rate and moderate genetic load, long-term population viability (probability of extinction $<0.01$ ) was only achieved with $\geq 30$ females and $\geq 3$ males released. To maintain $>95 \%$ genetic diversity of the source population in an isolated population, 50 females and 5 males are recommended to compose the founding population. Sensitivity analyses revealed first-year survival and reproductive rate were the simulation parameters with the greatest proportional influence on probability of extinction and genetic diversity. These simulations highlight important considerations for translocation success and data gaps including true genetic load in wild giraffe populations.
\end{abstract}

KEY WORDS: Founding population $\cdot$ Giraffe $\cdot$ Population model $\cdot$ PVA $\cdot$ Translocation $\cdot$ Population viability

\section{INTRODUCTION}

Giraffes Giraffa camelopardalis Linnaeus are endemic African ruminant ungulates and one of only a handful of extant terrestrial megaherbivores (OwenSmith 1988). Most populations of giraffes have declined in recent decades, leading to the recent decision

\footnotetext{
${ }^{*}$ Corresponding author: dereklee@psu.edu
}

to upgrade the species to Vulnerable and some subspecies as Endangered on the IUCN Red List (Muller et al. 2018). Translocations have been used as a conservation tool to re-introduce giraffes to previously occupied areas or establish new populations (Winter et al. 2019), but quantitative guidelines for establishing viable populations of translocated giraffes do not exist.

(C) The authors 2020. Open Access under Creative Commons by Attribution Licence. Use, distribution and reproduction are unrestricted. Authors and original publication must be credited. 
General guidelines for translocations suggest population modelling should be used to assist in the assessment of project feasibility (IUCN 2013). Population modelling for translocation projects can offer guidance on age- and sex-classes for founder populations by providing estimates of population persistence, long-term viability, and genetic diversity (Seddon et al. 2007). Population viability analysis (PVA) is commonly used to predict the likely future status of a population and thus offers a quantitative basis for evaluating alternative management strategies (Boyce 1992, Morris \& Doak 2002). The availability of software programs such as Vortex has facilitated the application of population modelling in re-introduction planning (e.g. Bustamante 1996) and postrelease evaluation (e.g. Slotta-Bachmayr et al. 2004).

Our objective was to identify the minimum size and sex ratio of a founding population of giraffes that would ensure long-term population viability and genetic diversity. We also present this as an example of how translocation planning can be improved using a quantitative approach. We simulated various scenarios of translocated giraffe populations using PVA implemented in Vortex 10 software (Lacy \& Pollak 2018). We used stochastic, single-population, individual-based models to project future population trajectories and estimate probability of extinction (PE), stochastic rate of population increase $(r)$, and genetic diversity (GD) at a hypothetically ideal prospective release site under different founder population release scenarios. Scenarios varied the numbers of females and males to release (Converse et al. 2013) and included 3 levels of inbreeding genetic load under demographic rates simulating slow, moderate, and fast population growth rates. We considered 4 criteria for defining successful translocations: $100 \mathrm{yr}$ probability of extinction $(\mathrm{PE})<0.05$ and $\mathrm{PE}<0.01$ (Morris \& Doak 2002) and 100 yr genetic diversity (GD) $>80 \%$ and GD $>95 \%$ of the source population (Frankham et al. 2010). The time frame of $100 \mathrm{yr}$ was selected because preliminary analyses revealed that transient population dynamics during the early decades postrelease showed positive population growth that was inevitably lost in later decades in smaller founding populations as stable age distribution and inbreeding effects resulted in non-viable populations. Therefore, we used the $100 \mathrm{yr}$ timespan because that duration is required to determine whether a translocation is truly successful in terms of long-term viability. We also performed a sensitivity analysis to determine which demographic and genetic load parameters had the greatest impact on translocation success, measured as proportional influence on PE and GD.

\section{MATERIALS AND METHODS}

We constructed population models in the program Vortex to simulate a hypothetical translocated population of simulated individuals using demographic parameters from the literature (Dagg 2014, Lee \& Strauss 2016) and publications of the Giraffe International Studbook (Bingaman Lackey 2009). In our models, males bred from ages 2 to 25 and females from ages 3 to 29; the maximum age of survival for both sexes was $30 \mathrm{yr}$, females always produced 1 calf, and sex ratio at birth was equal (Dagg 2014). Within the model parameters, offspring were dependent upon their mother for $1 \mathrm{yr}$, meaning that if the mother died during the calf's first year, then the calf also died (Dagg 2014). All males aged 2 and above were in the breeding pool. Demographic rates are given in Table 1.

To parameterize our PVA, we based our demographic rates on published observations of means and variances of age-specific survival and fecundity for wild giraffe populations throughout Africa (Lee \& Strauss 2016) and on data for reproductive longevity and inbreeding depression from the global zoo population (Bingaman Lackey 2009). We used data from IUCN Red List assessments to compute mean population growth rates for 7 growing giraffe subpopulations, including some translocations (Muller et al. 2018). The mean observed population growth rate was 1.024 (range 1.0045 to 1.035). Because the highest observed growth rates were from short time spans, they were likely due to transient population dynamics and not the asymptotic growth rate. Therefore, in our PVA, we simulated populations with 3 different demographic parameterizations with different asymptotic population growth rates $(\lambda)$ that represented slow- (1.001), moderate- (1.010), and fastgrowing (1.024) populations (Lee \& Strauss 2016, Muller et al. 2018). The fastest population growth rate is probably only achievable in translocation des-

Table 1. Demographic rates and variability (SD: standard deviation) used to parameterize population simulations of giraffe translocations reflecting slow, moderate, and fast population growth rates

\begin{tabular}{|c|c|c|c|c|c|c|}
\hline \multirow{2}{*}{$\begin{array}{l}\text { Population growth rate } \\
\lambda \\
\text { Demographic rates }\end{array}$} & \multicolumn{2}{|c|}{$\begin{array}{l}\text { Slow } \\
1.001\end{array}$} & \multicolumn{2}{|c|}{$\begin{array}{c}\text { Moderate } \\
1.010\end{array}$} & \multicolumn{2}{|c|}{$\begin{array}{l}\text { Fast } \\
1.024\end{array}$} \\
\hline & $\%$ & $\mathrm{SD}$ & $\%$ & $\mathrm{SD}$ & $\%$ & $\mathrm{SD}$ \\
\hline Mortality Age 0-1 & 50 & 10 & 50 & 10 & 40 & 10 \\
\hline Mortality Age 1-2 & 20 & 6 & 20 & 6 & 20 & 6 \\
\hline Mortality Age $>2$ & 5 & 3 & 5 & 3 & 5 & 3 \\
\hline Reproduction & 45 & 10 & 50 & 10 & 50 & 10 \\
\hline
\end{tabular}


tinations that have few or no large predators (Lee \& Strauss 2016, Muller et al. 2018).

Given that typically few individuals are translocated on any given occasion, demographic stochasticity, Allee effects, and inbreeding depression could all adversely affect long-term viability for translocated populations (O'Grady et al. 2006, Deredec \& Courchamp 2007, Van Houtan et al. 2009). We included stochasticity (demographic rates vary among individuals and over time) and inbreeding depression (reduced reproduction and calf survival due to inbreeding) in our simulations because these effects are likely to exist in giraffe populations (O'Grady et al. 2006, Bingaman Lackey 2009, Lee \& Strauss 2016, Lacy et al. 2018). We did not include Allee effects (reduced demographic rates at small population size) because Allee effects are unlikely in giraffe populations, especially in enclosed reserves where mates can easily locate each other.

We included 3 levels of inbreeding depression. Using data from the giraffe studbook, we estimated the minimum genetic load as the number of total lethal equivalents (LE) per individual with a regression analysis of natural-log-transformed first-year survival on F-coefficient according to Morton et al. (1956). The slope of the regression provided an estimate of the reduction of fitness due to inbreeding and gave the lower bound approximation of the effective number of LE per gamete, so we doubled the slope to estimate the low value for number of LE per zygote (Lynch \& Walsh 1998). We used Vortex inbreeding values representing a low genetic load of $2.5 \mathrm{LE}$ per individual (from our regression), a moderate load of 6.29 LE (O'Grady et al. 2006, Nietlisbach et al. 2019), and a high genetic load of 12.6 LE (double the moderate load). Fifty percent of LE are due to recessive lethal alleles. The other half of the LE are due to overdominance (heterozygote superiority), and this genetic load cannot be removed by selection (Lacy et al. 2018). We set demographic temporal correlation between mortality and reproduction due to environmental variation at 0.5 . The values for percent LE due to recessive lethal alleles and temporal correlation were the default values of Vortex (Lacy et al. 2018).

We assumed zero translocation-related mortality, so no additional mortality effect above normal levels due to the process of capturing, relocating, and releasing. If mortality is expected during the translocation process, our simulation results should be interpreted using the actual number of successful live releases. We assumed zero post-release dispersal movements because many translocations will likely be into fenced or otherwise constrained areas and because we wanted to keep track of every individual in the translocated population.

We projected 198 PVA scenarios. We simulated populations with various numbers of 2 yr old females released $(5,10,20,30,40,50,60$, and 80 females $)$ and different numbers of 2 yr old males released to vary the sex ratio $(\mathrm{SR})$ at release $(\mathrm{SR}=$ males/females, range $=0.1$ to 0.5 ). We simulated each combination of number of females and males at the 3 levels of asymptotic growth rate $(\lambda)$ and 3 levels of genetic load.

For all scenarios, we ran 1000 iterations of our stochastic model to project the populations for $100 \mathrm{yr}$. We selected a projection time of 100 yr because giraffes are a long-lived species and we were most interested in the longer-term implications of translocation decisions, particularly the effect of the number of individuals released. The extinction definition was $\mathrm{N}<2$. We assumed a hypothetically ideal release site but included effects of density dependence with a carrying capacity of $\mathrm{N}=1000$. This was done because we were primarily interested in the effects of demography and genetics without the confounding effects of habitat limitation, which varies considerably between translocation sites. Forage quality and availability in all seasons should be quantified at the release site to determine carrying capacity as part of a comprehensive pre-translocation assessment. For each scenario, we recorded output on PE and GD (as observed heterozygosity) in the final extant population. We provide guidelines for founding population size and sex ratio for successful translocations with success defined 4 ways: $\mathrm{PE}<0.05$ and $\mathrm{PE}<0.01$; and GD $>80 \%$ and GD $>90 \%$.

We performed a sensitivity analysis to determine which demographic or inbreeding parameter was most influential to long-term viability by comparing outcomes from simulations that were identical in every way except that we reduced a single demographic or inbreeding parameter by $\sim 25 \%$. We simulated release of 30 females and 3 males using moderate demographic rates (Table 1, middle columns) and moderate genetic load ( $\mathrm{LE}=6.29$ ) as the reference simulation. We then simulated 6 populations identical except for a single change: (1) increased age of first reproduction by 1 yr for males and females; (2) first-year mortality $=63 \%$; (3) second-year mortality $=25 \%$; (4) adult mortality $=6 \%$; (5) reproduction = $38 \%$; and (6) LE = 7.9. The observed differences in $\mathrm{PE}$ and GD between the changed simulations and the reference simulation indicate the sensitivity due to a proportionally similar change in each demographic or genetic load parameter. 


\section{RESULTS}

Population projections from all our scenarios followed the same general post-release trajectory. Overall, there was an initial population decline for $1 \mathrm{yr}$ as some released individuals died before maturity, followed by $27 \mathrm{yr}$ of rapid population growth as the founders and their offspring reproduced, then a $1 \mathrm{yr}$ decline in Year 29 as the last remaining founding animals died. This was followed by a brief $3 \mathrm{yr}$ period of stable or positive population growth until the first post-founder generation died and stable age distribution was achieved. Long-term (100 yr) viability depended on asymptotic population growth rate and inbreeding genetic load, as well as which success criteria were used, but the minimum founding population varied from 10 to 60 females and 1 to 18 males (Table 2).

The 100 yr PE declined as the number of released females and sex ratio increased (Fig. 1). Assuming fast population growth demographic rates and minimum inbreeding genetic load, the 100 yr PE was $<0.05$ when release included at least 10 females and 3 males (Table 2). Under more conservative demographic rates $(\lambda=1.001)$ and realistic inbreeding genetic loads ( $\mathrm{LE}=6.3$ or 12.6), the minimum release population for $95 \%$ viability at $100 \mathrm{yr}$ was 30 females and 3 to 6 males (Table 2). Final GD increased with larger numbers of females and males in the founding population (Fig. 2). To meet the success criteria of preserving $80 \%$ of GD, minimum founding population sizes were similar to those required for the criteria of $\mathrm{PE}<0.05$, i.e. 30 females and 3 to 6 males (Table 2).

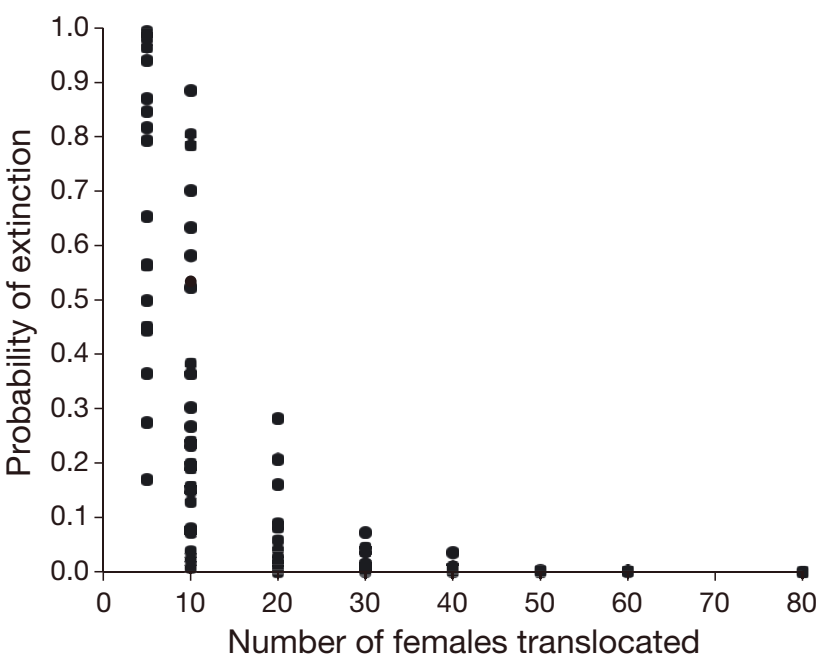

Fig. 1. The 100 yr probability of extinction decreased as the number of released females increased in population projections simulating translocations of different numbers of wild giraffes. Simulations used population growth rates $(\lambda)$ of $1.001,1.010$, and 1.024 and genetic load as number of lethal equivalents (LE) of 2.5, 6.29, and 12.6

Sensitivity analysis revealed demographic parameters of first-year survival and adult female reproductive rate were the most influential on long-term viability metrics of PE and GD (Table 3). Changes in first-year mortality and reproductive rate both increased PE $>10 \%$ and reduced GD $>10 \%$. Increasing the age of first reproduction by $1 \mathrm{yr}$ resulted in a $1 \%$ increase in $\mathrm{PE}$ and $2 \%$ reduction in $\mathrm{GD}$, and all other parameter changes resulted in $<1 \%$ reduction in $\mathrm{PE}$ and $\leq 2 \%$ reduction in GD (Table 3 ).
Table 2. Minimum founding population sizes of female (F) and male (M) giraffes for translocations to achieve $100 \mathrm{yr}$ population viability success under 4 criteria of success, with 3 levels of intrinsic population growth rate and 3 levels of inbreeding genetic load. LE: lethal equivalents

\begin{tabular}{|c|c|c|c|c|}
\hline Criteria & $\begin{array}{l}\text { Population } \\
\text { growth rate }\end{array}$ & $\begin{array}{r}\text { Inbre } \\
\text { Low (2.5 LE) }\end{array}$ & $\begin{array}{l}\text { eeding genetic } \\
\text { Mod (6.3 LE) }\end{array}$ & $\begin{array}{l}\text { load } \\
\text { High (12.6 LE) }\end{array}$ \\
\hline $\begin{array}{l}<0.05 \text { Probability } \\
\text { of extinction }\end{array}$ & $\begin{array}{l}\text { Slow (1.001) } \\
\text { Mod (1.010) } \\
\text { Fast }(1.024)\end{array}$ & $\begin{array}{l}20 F, 2 M \\
20 F, 2 M \\
10 F, 3 M\end{array}$ & $\begin{array}{l}30 F, 3 M \\
20 F, 2 M \\
10 F, 5 M\end{array}$ & $\begin{array}{l}30 \mathrm{~F}, 6 \mathrm{M} \\
30 \mathrm{~F}, 3 \mathrm{M} \\
20 \mathrm{~F}, 2 \mathrm{M}\end{array}$ \\
\hline $\begin{array}{l}<0.01 \text { Probability } \\
\text { of extinction }\end{array}$ & $\begin{array}{l}\text { Slow (1.001) } \\
\text { Mod (1.010) } \\
\text { Fast }(1.024)\end{array}$ & $\begin{array}{l}30 F, 3 M \\
20 F, 2 M \\
10 F, 5 M\end{array}$ & $\begin{array}{l}30 F, 6 M \\
30 F, 3 M \\
20 F, 2 M\end{array}$ & $\begin{array}{l}40 F, 5 M \\
30 F, 9 M \\
20 F, 5 M\end{array}$ \\
\hline $\begin{array}{l}>80 \% \text { Genetic } \\
\text { diversity }\end{array}$ & $\begin{array}{l}\text { Slow (1.001) } \\
\text { Mod (1.010) } \\
\text { Fast }(1.024)\end{array}$ & $\begin{array}{l}20 \mathrm{~F}, 2 \mathrm{M} \\
20 \mathrm{~F}, 2 \mathrm{M} \\
10 \mathrm{~F}, 1 \mathrm{M}\end{array}$ & $\begin{array}{l}20 F, 2 M \\
20 F, 2 M \\
10 F, 3 M\end{array}$ & $\begin{array}{l}30 F, 3 M \\
20 F, 2 M \\
20 F, 2 M\end{array}$ \\
\hline $\begin{array}{l}>95 \% \text { Genetic } \\
\text { diversity }\end{array}$ & $\begin{array}{l}\text { Slow (1.001) } \\
\text { Mod (1.010) } \\
\text { Fast (1.024) }\end{array}$ & $\begin{array}{l}50 \mathrm{~F}, 15 \mathrm{M} \\
50 \mathrm{~F}, 5 \mathrm{M} \\
40 \mathrm{~F}, 5 \mathrm{M}\end{array}$ & $\begin{array}{l}60 \mathrm{~F}, 12 \mathrm{M} \\
50 \mathrm{~F}, 5 \mathrm{M} \\
40 \mathrm{~F}, 5 \mathrm{M}\end{array}$ & $\begin{array}{c}60 \mathrm{~F}, 18 \mathrm{M} \\
50 \mathrm{~F}, 10 \mathrm{M} \\
40 \mathrm{~F}, 5 \mathrm{M}\end{array}$ \\
\hline
\end{tabular}

\section{DISCUSSION}

Despite the potentially low chance of success (Fischer \& Lindenmayer 2000), translocations continue to be used as a major tool in endangered species management. Our analyses evaluated the likely result of a giraffe translocation under reasonable demographic and genetic assumptions based on published literature and provided quantitative guidance to improve outcomes. We considered a population to be minimally demographically viable when it had a $\leq 5 \%$ probability of extinction during a 100 yr period and minimally genetically viable when it has a $\leq 20 \%$ 


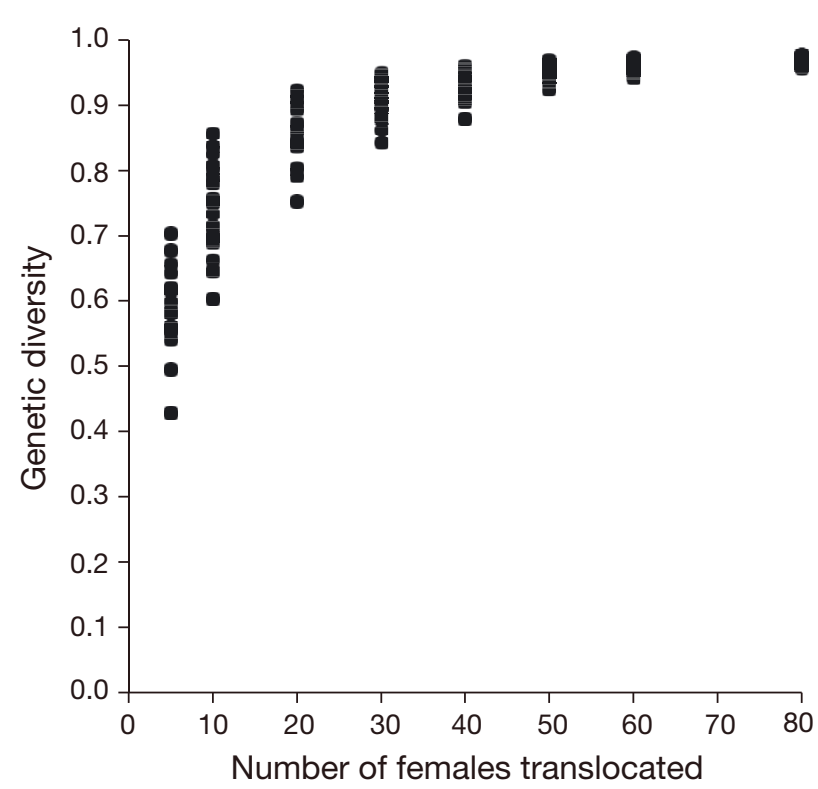

Fig. 2. Gene diversity in final extant populations increased as the number of released females increased in $100 \mathrm{yr}$ population projections simulating 198 translocations of wild giraffes. Simulations used $\lambda$ values of $1.001,1.010$, and 1.024 and lethal equivalent (LE) values of $2.5,6.29$, and 12.6

Table 3. Sensitivity analysis of demographic and genetic load parameters for simulations of giraffe translocations where each parameter was proportionally reduced $\sim 25 \%$. $\triangle \mathrm{PE}$ : change in probability of extinction relative to the reference simulation; $\triangle \mathrm{GD}$ : change in genetic diversity relative to the reference simulation

\begin{tabular}{|lccc|}
\hline Parameter & Value & $\Delta \mathrm{PE}$ & $\Delta \mathrm{GD}$ \\
\hline First-year mortality & $63 \%$ & 0.114 & -0.106 \\
Reproductive rate & $38 \%$ & 0.102 & -0.105 \\
Age of first reproduction & $\mathrm{M}=3, \mathrm{~F}=4$ & 0.009 & -0.024 \\
Second-year mortality & $25 \%$ & 0.002 & -0.020 \\
Adult mortality & $6 \%$ & 0.000 & -0.009 \\
Genetic load lethal & 7.90 & 0.003 & -0.006 \\
$\quad$ equivalents & & & \\
\hline
\end{tabular}

decrease in genetic diversity compared to the original wild population over the same period (IUCN 2013). These minimum criteria for viability of an isolated population were achieved when 20 females and 2 males were released, assuming demographic parameters for a moderate population growth rate $(\lambda=$ 1.010 ) and a moderate amount of inbreeding genetic load (lethal equivalents: $L E=6.29$ ). Under more conservative demographic rates and higher genetic loads, the minimum release population for $95 \%$ viability at $100 \mathrm{yr}$ was 30 females and 3 to 6 males. This latter scenario also managed to preserve at least $80 \%$ of the genetic diversity of the source population. Population projections from our scenarios illuminate the marked impact of asymptotic population growth and genetic load on the long-term viability and genetic diversity. This study highlights the data gaps remaining, the sensitivity of translocation success to variation in population modeling parameters, and the need for careful project-specific monitoring and analyses.

Few studies have thoroughly monitored source and translocated populations for changes in genetic parameters such as heterozygosity, allelic richness, and the rate or level of inbreeding (Groombridge et al. 2012, Puckett et al. 2014). Ideally, genetic variability should be assessed in source populations in advance of translocations to use genetic information to guide translocation plans and to provide a contrast to posttranslocation genetic studies (Rocamora \& Richardson 2003, Biebach \& Keller 2010). This is particularly important if there is reason to assume that the source population already has low genetic diversity, for example, because it was established through translocations or because it was a long-isolated population of only a few hundred individuals. Consideration should also be given to the issues that can arise when using individuals for reintroduction from captive stocks where genetic adaptation to captivity may have occurred (Montgomery et al. 2010, Robert 2009), which becomes more likely with more generations in captivity but should have been partially mitigated if the population was managed to minimize inbreeding (Frankham et al. 2010, Marsden et al. 2013).

During the 'founders' years' from Year 2 to 23 in all our projections, initial observed population growth will likely be positive if $\geq 2$ females are translocated. However, this initial growth is a transient effect of the young-skewed age distribution of the founding population, and very few translocations of small $<<20$ females) founding populations will be viable in the long term in isolation due to a lack of genetic diversity (Fig. 1). Increasing the number of females in the initial release had a strong positive effect on mean annual stochastic rate of population increase, and final genetic diversity. A 0.01 probability of extinction was achieved assuming moderate demographic rates and moderate genetic load if the founder population included 30 females and 3 males. In situations where the translocation destination has few or no large predators, and asymptotic growth rates could approach 1.024, then viable populations could be achieved with releases of a minimum of 20 females and 2 males that are all unrelated. These minimum founding populations will result in a $20 \%$ loss of genetic diversity, so if genetic diversity considera- 
tions are paramount and additional translocations at a later stage are unlikely, then much larger founding populations should be attempted.

Note that the low final genetic diversity is not due to a lack of genetic diversity in the founding population but due to the rapid loss of genetic diversity in the first years when the population is still small. A population that is twice as large loses genetic diversity at half the speed. This highlights the importance of ensuring that a reintroduced population is as large as possible at founding and can grow as fast as possible in this first stage. Population growth could be facilitated, for example, by providing supplemental water or food, or veterinary care, especially for individuals with under-represented alleles. This may seem to counteract natural selection, but selection works inefficiently on small populations (Margan et al. 1998). Mitigating the loss of genetic diversity due to stochastic effects in the short-term maximizes the genetic material available to a population when it has grown large enough for functional natural selection, which is several hundreds of individuals. Also, the loss of genetic diversity can be mitigated by immigration of a few individuals from another population every generation, with the exact number of migrants being dependent on the situation (Wang 2004, Puckett et al. 2014). If there are natural corridors, this exchange may happen naturally. For fenced or isolated areas, this will require periodic translocations of individuals. Populations established with a low number of founders (e.g. Muller 2019, Table 3) may still be useful from a species conservation perspective; however, additional gene flow will need to be facilitated at some point in the following decades. Any translocation also carries the risk of spreading diseases.

Rules of thumb for translocations such as those presented here should not be used as a substitute for project-specific monitoring and analyses, but they can be useful in early decision-making and feasibility analyses. We recognize that the $100 \mathrm{yr}$ timespan of our study is long, and numerous stochastic factors such as anthropogenic modification of habitats and climate change can influence demographic rates during that period. Ideally, every translocation should be conducted under an adaptive management framework with continuous pre- and post-translocation monitoring of both the source and destination populations and annual updates to PVAs to inform structured decision-making (McCarthy et al. 2012, Williams \& Brown 2014). The use of continuous monitoring, formal and transparent decision-making, and data-driven adaptive management cycles will gener- ate the necessary data to estimate demographic parameters, identify prospective subject animals, predict population viability, and make informed management decisions such as whether to augment. The true demographic rates of any source and translocated population should be estimated annually and compared with assumptions made during planning. As our sensitivity analysis showed, if demographic rates such as juvenile survival or reproductive rates are below critical levels, the translocated population will be considerably less viable than was predicted.

The ideal female age class for translocations in terms of population growth rate is the youngest age class that can immediately begin reproducing, but adult giraffes are logistically challenging to move due to their large body size. All of our population projections used the logistically more tractable age class of 2 yr olds for translocation, as this age class is often chosen for translocations. Translocating juveniles means there is an inevitable initial post-release decline in population size as animals are subject to mortality before reaching reproductive age. Sensitivity analyses indicated that ages of first reproduction above our very optimistic values of 3 for females and 2 for males can adversely affect the rate of population increase and long-term viability.

Fewer males than females are necessary in translocations because the male contribution to population viability is mostly confined to their genetic contributions, and equal offspring sex ratios will greatly increase the number of adult males within 1 generation. Our projections assumed all males were in the breeding pool and had equal probability of mating. The details of giraffe mating success are largely unknown for wild populations, but there is evidence that dominant males may monopolize mating opportunities, potentially resulting in a skewed mating success among existing males (Pratt \& Anderson 1982, 1985). Similarly, female mate choice could result in a skewed mating success among males (Andersson 1994). Either of these situations would reduce the pool of breeding males, thereby reducing the genetic diversity passed to subsequent generations, leading to a smaller effective population size, lower genetic diversity, and possibly higher inbreeding depression.

There are many additional challenges to translocations at the initial stages beyond maintaining genetic diversity in the long term. For example, managers considering translocations are faced with a trade-off between either moving individuals found together in herds to maintain social structure, as closely associ- 
ated female giraffes tended to be more related than expected by chance (Carter et al. 2013), or moving non-related individuals to maintain genetic diversity in the founder population. Other concerns include depletion of genetic diversity and population viability of the source population if it is small and moving individuals into artificial situations where they are no longer subject to natural processes. These concerns are beyond the scope of this paper but are addressed elsewhere (Muller et al. in press).

Future research to inform translocation PVAs should focus on 2 critical data gaps. First, research should aim to quantify inbreeding depression in wild or semi-wild populations that have and have not experienced a population bottleneck and to quantify genetic parameters in translocated versus source populations over time. Second, research should determine whether assumptions of panmictic breeding and equal breeding success of all males are accurate for small giraffe populations. This is important because skewed mating success will reduce the effective population size and worsen the impact of inbreeding, i.e. result in more severe inbreeding depression. Published accounts of translocation outcomes including annual demographic rates of age-specific survival and reproduction, genetic diversity, and long-term population viability are also needed as giraffe translocations are typically not well documented (Winter et al. 2019). It is unlikely that this specific PVA will apply to other species of ungulate due to giraffe's unusual delayed maturity and slow reproduction rate. However, the results of this study do apply conceptually to the establishment of populations of other species with a slow population growth rate. Nevertheless, the approach of our study can be adopted by planners as a template for the PVA in translocations of other ungulates, using appropriate species-specific parameterization of the Vortex model.

Acknowledgements. We thank 3 anonymous reviewers and the handling editor who improved this manuscript.

\section{LITERATURE CITED}

Andersson M (1994) Sexual selection. Princeton University Press, Princeton, NJ

Biebach I, Keller LF (2010) Inbreeding in reintroduced populations: the effects of early reintroduction history and contemporary processes. Conserv Genet 11:527-538

Bingaman Lackey LB (2009) Giraffe studbook Giraffa camelopardalis North American Regional/ Global. Association of Zoos and Aquariums and Disney's Animal Kingdom, Orlando, FL

Boyce MS (1992) Population viability analysis. Annu Rev Ecol Syst 23:481-497
Bustamante J (1996) Population viability analysis of captive and released bearded vulture populations. Conserv Biol 10:822-831

* Carter KD, Seddon JM, Frère CH, Carter JK, Goldizen AW (2013) Fission-fusion dynamics in wild giraffes may be driven by kinship, spatial overlap and individual social preferences. Anim Behav 85:385-394

Converse SJ, Moore CT, Armstrong DP (2013) Demographics of reintroduced populations: estimation, modeling, and decision analysis. J Wildl Manag 77:1081-1093

Dagg AI (2014) Giraffe: biology, behaviour and conservation. Cambridge University Press, Cambridge

* Deredec A, Courchamp F (2007) Importance of the Allee effect for reintroductions. Ecoscience 14:440-451

*Fischer J, Lindenmayer DB (2000) An assessment of the published results of animal relocations. Biol Conserv 96: $1-11$

Frankham R, Ballou JD, Briscoe DA (2010) Introduction to conservation genetics. Cambridge University Press, Cambridge

Groombridge JJ, Raisin C, Bristol R, Richardson DS (2012) Genetic consequences of reintroductions and insights from population history. In: Ewen JG, Armstrong DP, Parker KA, Seddon PJ (eds) Reintroduction biology: integrating science and management. Wiley-Blackwell, Chichester, p 395-440

International Union for the Conservation of Nature (IUCN) (2013) Guidelines for reintroductions and other conservation translocations. IUCN SSC Re-Introduction Specialist Group, Gland

Lacy RC, Pollak JP (2018) VORTEX: a stochastic simulation of the extinction process. Chicago Zoological Society, Brookfield, IL

Lacy RC, Miller PS, Traylor-Holzer K (2018) Vortex 10 user's manual. 1 June 2018 update. IUCN SSC Conservation Breeding Specialist Group, Gland, and Chicago Zoological Society, Apple Valley, MN

KLee DE, Strauss MKL (2016) Giraffe demography and population ecology. Reference Module in Earth Systems and Environmental Sciences. Elsevier, Amsterdam, available via ScienceDirect

Lynch M, Walsh B (1998) Genetics and analysis of quantitative traits. Sinauer, Sunderland, MA

* Margan SH, Nurthen RK, Montgomery ME, Woodworth LM, Lowe EH, Briscoe DA, Frankham R (1998) Single large or several small? Population fragmentation in the captive management of endangered species. Zoo Biol 17: 467-480

Marsden CD, Verberkmoes H, Thomas R, Wayne RK, Mable BK (2013) Pedigrees, MHC and microsatellites: an integrated approach for genetic management of captive African wild dogs (Lycaon pictus). Conserv Genet 14: 171-183

McCarthy MA, Armstrong DP, Runge MC (2012) Adaptive management of reintroductions. In: Ewen JG, Armstrong DP, Parker KA, Seddon PJ (eds) Reintroduction biology, integrating science and management. Wiley-Blackwell, Chichester, p 256-289

Montgomery ME, Woodworth LM, England PR, Briscoe DA, Frankham R (2010) Widespread selective sweeps affecting microsatellites in Drosophila populations adapting to captivity: implications for captive breeding programs. Biol Conserv 143:1842-1849

Morris WF, Doak DF (2002) Quantitative conservation biology. Sinauer, Sunderland, MA 
Morton NE, Crow JF, Muller HJ (1956) An estimate of the mutational damage in man from data on consanguineous matings. Proc Natl Acad Sci USA 42:855-863

Muller Z (2019) Rothschild's giraffe Giraffa camelopardalis rothschildi (Linnaeus, 1758) in East Africa: a review of population trends, taxonomy and conservation status. Afr J Ecol 57(1):20-30

Muller Z, Bercovitch F, Brand R, Brown D and others (2018) Giraffa camelopardalis (amended version of 2016 assessment) Gland, Switzerland. The IUCN Red List of Threatened Species 2018:e.T9194A136266699

Muller Z, Lee DE, Scheijen C, Strauss MKL, Carter KD, Deacon F (in press) Giraffe translocations: recommendations to improve outcomes. J Afr Ecol

Nietlisbach P, Muff S, Reid JM, Whitlock MC, Keller LF (2019) Nonequivalent lethal equivalents: models and inbreeding metrics for unbiased estimation of inbreeding load. Evol Appl 12:266-279

O'Grady JJ, Brook BW, Reed DH, Ballou JD, Tonkyn DW, Frankham R (2006) Realistic levels of inbreeding depression strongly affect extinction risk in wild populations. Biol Conserv 133:42-51

Owen-Smith RN (1988) Megaherbivores: the influence of very large body size on ecology. Cambridge University Press, New York, NY

Pratt DM, Anderson VH (1982) Population, distribution, and behaviour of giraffe in the Arusha National Park, Tanzania. J Nat Hist 16:481-489

Pratt DM, Anderson VH (1985) Giraffe social behaviour. J Nat Hist 19:771-781

Editorial responsibility: Matt Hayward, Callaghan, New South Wales, Australia
Puckett EE, Kristensen TV, Wilton CM, Lyda SB and others (2014) Influence of drift and admixture on population structure of American black bears (Ursus americanus) in the central interior highlands, USA, 50 years after translocation. Mol Ecol 23:2414-2427

Kobert A (2009) Captive breeding genetics and reintroduction success. Biol Conserv 142:2915-2922

Kocamora G, Richardson DS (2003) Genetic and morphological differentiation between the two remnant populations of Seychelles white-eye Zosterops modestus. Ibis 145: $34-44$

Seddon PJ, Armstrong DP, Maloney RF (2007) Developing the science of reintroduction biology. Conserv Biol 21:303-312

* Slotta-Bachmayr L, Boegel R, Kaczensky P, Stauffer C, Walzer C (2004) Use of population viability analysis to identify management priorities and success in reintroducing Przewalski's horses to southwestern Mongolia. J Wildl Manag 68:790-798

* Van Houtan KS, Halley JM, Van Aarde R, Pimm SL (2009) Achieving success with small, translocated mammal populations. Conserv Lett 2:254-262

* Wang J (2004) Application of the one migrant per generation rule to conservation and management. Conserv Biol 18:332-343

* Williams BK, Brown ED (2014) Adaptive management: from more talk to real action. Environ Manage 53:465-479

* Winter S, Coimbra RTF, Bronec A, Hay C, Salb AL, Fennessy J, Janke A (2019) Species assignment and conservation genetics of giraffe in the Republic of Malawi. Conserv Genet 20:665-670

Submitted: October 8, 2019; Accepted: December 13, 2019 Proofs received from author(s): January 25, 2020 